Neto, O. L. M., Malta, D. C., Mascarenhas, M. D. M., Duarte, E. C., Silva, M. M. A., Oliveira, K. B.,... Porto, D. L. (2010). Fatores de risco para acidentes de transporte terrestre entre adolescentes no Brasil: Pesquisa $\mathrm{Na}$ cional de Saúde do Escolar (PeNSE). Ciência \& Saúde Coletiva, 15(Supl. 2), 3043-3052. doi:10.1590/S141381232010000800009

Organização das Nações Unidas. (2015). ONU: Acidentes no trânsito ainda matam 1,25 milhão por ano, 90\% em países de renda média e baixa. Disponivel em https://nacoesunidas.org/onu-acidentes-no-transito-ainda-matam-125-milhao-por-ano-90-em-paises-de-renda-media-e-baixa/

Ministérios da Sáde. (2015). Pesquisa Nacional de Saúde 2013: Acesso e utilização dos serviços de saúde, acidentes e violências. Brasil, grandes regiões e unidades da federação. Rio de Janeiro: Instituto Brasileiro de Geografia e Estatística, Coordenação de Trabalho e Rendimento. Disponivel em biblioteca.ibge.gov.br/ visualizacao/livros/liv94074.pdf

Peuker, A. C., Fogaça, J., \& Bizarro, J. L. (2006). Expectativas e beber problemático entre pectativas e be rios. Psicologian. Teoria e Pesquisa, 22(2).

Silva, D. P., Barbosa, M. H., \& Chavaglia, S. R. R. (2010) Utilização de equipamentos de segurança entre vítimas de acidentes no município de Uberaba-MG. Revista Eletrônica de Enfermagem,12(1), 83-88. doi:10.5216/ree.v12i1.5482.

Silva, R. A., Jansen, K., Godoy, R. V., Souza, L. D. M., Horta, B. L., \& Pinheiro, R. T. (2009). Prevalência e fatores associados a porte de arma e envolvimento em agressão física entre adolescentes de 15 a 18 anos: Estudo de base populacional. Cadernos de Saúde Pública, 25(12), 2737-2745. doi:10.1590/s0102-311×2009001200020
AUTORES:

Ivanete Fernandes do Prado ${ }^{1,2}$

Berta Leni Costa Cardoso ${ }^{1}$

Elionara Teixeira Boa Sorte ${ }^{1}$

Marcela Andrade Rios

Nanci Maria de França ${ }^{2}$

Universidade do Estado da Bahie UNEB, Brasil

Universidade Católica de Brasília,

UCB, Brasil

https://doi.org/10.5628/rpcd.17 S3A.65
Hipertensão arterial

\section{durante a gravidez}

\section{PALAVRAS CHAVE:}

Síndromes hipertensivas.

Prematuridade. Baixo peso ao nascer.

Promoção de saúde. Exercício físico.

\title{
RESUMO
}

Este estudo objetivou identificar a prevalência de gestantes com a hipertensão arterial durante a internação para o parto e associá-la com a prematuridade e o baixo peso ao nascer. Trata-se de estudo com delineamento tipo caso controle no qual as associações das variáveis preditoras com desfecho prematuridade e baixo peso ao nascer foram analisadas por meio de modelo hierarquizado de regressão logística múltipla. A prevalência de gestantes com hipertensão arterial durante a internação para o parto na amostra estudada foi de $13.5 \%$. Foram observadas associações significativas da hipertensão arterial tanto com a prematuridade, quanto com o baixo peso ao nascer. Gestantes com hipertensão apresentam 3.47 (IC95\%: 1.37 - 8.81) vezes mais chance de ter filho prematuro e 2.55 (1.03 6.32) vezes mais chance de ter filho com baixo peso do que aquelas sem fatores de risco à gestação. Gestantes hipertensas devem ser encaminhadas e asseguradas no serviço de acompanhamento de alto risco, bem como orientadas sobre a prática de exercícios físicos com o intuito de diminuir as complicações perinatais e maternas. 


\section{ABSTRACT}

This study aimed to identify the prevalence of pregnant women with hypertension during hospitalization for childbirth and analyze its associating with prematurity and low birth weight. This is case-control study in which the associations of the predictor variables with the outcome of prematurity and low birth weight were analyzed using hierarchical a model of multiple logistic regression. The prevalence of pregnant women with hypertension during hospitalization for childbirth in the sample was $13.5 \%$. Significant associations of hypertension were observed with either prematurity and with low birth weight. Pregnant women with hypertension are 3.47 (CI 95\%: 1.37 to 8.81 ) times more likely to have premature children and 2.55 (1.03 to 6.32) times more likely to have children with low birth weight than those without risk factors. Hypertensive pregnant women must be supervised and secured in high-risk monitoring services, and oriented on physical exercise in order to reduce perinatal and maternal complications.

\section{KEY-WORDS}

Hypertensive syndromes. Prematurity.

Low birth weight. Health promotion. Physical exercise.
A gravidez é um evento fisiológico sem maiores problemas para a maior parte das mulheres. No entanto, para algumas pode ocorrer injúria na evolução da gravidez, colocando em risco a saúde da mãe e do feto. A hipertensão arterial durante esse período é um exemplo de injúria que pode culminar na prematuridade e baixo peso ao nascer.

Essa condição clínica faz parte dos quatro tipos de síndromes hipertensivas da gravidez que podem ser classificadas como hipertensão crônica (é aquela observada antes da gravidez, antes de 20 semanas de gestação, ou diagnosticada pela primeira vez durante a gravidez e que não se resolve até 12 semanas após o parto), pré-eclâmpsia/ eclampsia (hipertensão que ocorre após 20 semanas de gestação acompanhada de proteinúria, com desaparecimento até 12 semanas pós-parto), eclampsia (caracteriza-se pela presença de convulsões tônico-clônicas generalizadas ou coma em mulher com qualquer quadro hipertensivo, não causadas por epilepsia ou qualquer outra doença convulsiva), pré-eclâmpsia sobreposta à hipertensão crônica (é o surgimento de pré-eclâmpsia em mulheres com hipertensão crônica ou doença renal) (Ministério da Saúde, 2012a).

As síndromes hipertensivas da gravidez podem desencadear danos à mãe e ao feto. Para a mulher, essas síndromes são a principal causa de morte materna, além de provocar várias complicações, como encefalopatia hipertensiva, falência cardíaca, grave comprometimento da função renal, hemorragia retiniana, coagulopatias e associação com pré-eclâmpsia. Para o feto essa situação de risco o predispõe a restrição do crescimento intrauterino, deslocamento prematuro de placenta, sofrimento fetal, morte intraútero, prematuridade e baixo peso ao nascer (Vettore, Dias, Madeira, Vettore, \& Leal, 2011). Como consequência, esses fatores, isolados ou em associação, culminam com o maior risco de morrer no primeiro ano de vida, com problemas de desenvolvimento na infância e com a maior probabilidade de ocorrência de várias doenças na vida adulta (Costa, Tibúrcio, Oliveira, Gontijo, \& Guimarães, 2014).

Conhecer e compreender o complexo processo que interfere na evolução da gravidez, incluindo aquele que aumenta os riscos do parto, poderá ser de grande utilidade para as políticas públicas de promoção da saúde e, consequentemente, melhoria da qualidade de serviços ofertados ao binômio mãe/ filho. Além disso, os achados têm implicação para a atuação das equipes de saúde, seja da atenção primária, secundária ou terciária, uma vez que muitos são os desfechos desfavoráveis para a mãe e o feto. Finalmente, outro desdobramento será a continuidade da pesquisa com as mesmas crianças em idade pré-escolar para avaliação da qualidade de vida, do desenvolvimento motor (fino e geral), psicossocial e da linguagem, o que implica um outro nível de abordagem para o público materno-infantil; estamos falando da educação/ promoção de saúde, uma vez que medidas não farmacológicas também são indicadas, como os exercícios físicos, que podem auxiliar a prevenção das síndromes hipertensivas.

Com base no exposto, objetiva-se neste estudo identificar a prevalência de gestantes com hipertensão arterial durante a internação para o parto e associá-la com a prematuridade e o baixo peso ao nascer. 
Trata-se de estudo com delineamento tipo caso-controle realizado no hospital público referência para gestação de alto risco, localizado no município de Guanambi Bahia, utilizando as declarações de nascidos vivos e prontuários das gestantes, relacionados aos partos ocorridos no período de janeiro a dezembro de 2011.

Foi escolhido o ano de 2011 para seleção da população porque este estudo faz parte de um projeto mais amplo em que as crianças nascidas prematuras e ou com baixo peso nesse período serão avaliadas quanto ao desenvolvimento e a qualidade de vida durante a idade pré-escolar. 0 projeto-mãe teve aprovação do Comitê de Ética em Pesquisa da Universidade Católica de Brasília e Secretaria de Saúde do Estado da Bahia sob o parecer no 913.952; de acordo com a resolução no 466, de 12 de dezembro de 2012, do Conselho Nacional de Saúde, que trata das Diretrizes e Normas da Pesquisa com Seres Humanos.

O município de Guanambi é referência em saúde para os municípios que compõem sua microrregião, sendo esse hospital público o principal estabelecimento de saúde que atende essa demanda. Administrado pelo Governo do Estado, o hospital integra a rede do Sistema Único de Saúde e comporta a demanda espontânea e referenciada, podendo ser atendimento ambulatorial, internação, serviço de apoio diagnóstico, terapêutica e urgência e contando om leitos cirúrgico, clínico, obstétrico, pediátrico, UTI neonatal e adultos (Ministério da Saúde, 2014).

\section{AMOSTRA}

Participaram 207 gestantes e 212 recém-nascidos vivos, sendo 125 gestantes e 128 recém-nascidos prematuros (idade gestacional menor que ou igual a 37 semanas) e/ ou com baixo peso (peso ao nascer menor que 2.500 gramas), que compuseram o grupo-caso; além disso, 93 gestantes e 95 recém-nascidos vivos com idade gestacional maior que 37 semanas e peso ao nascer maior que 2.500 gramas compuseram o grupo-controle. A diferença entre a quantidade de gestantes e a de recém-nascidos justifica-se pela ocorrência de 05 gestações duplas, sendo 03 no grupo-caso e 02 no controle.

INSTRUMENTOS

Para a coleta dos dados das Declarações de Nascidos Vivos e prontuários das gestantes fo utilizado um protocolo de pesquisa composto pelos dados do recém-nascido (sexo, peso Apgar e anomalias congênitas), dados mãe (idade, situação conjugal, raça, escolaridade e ocupação), gestação (idade gestacional, consultas de pré-natal e tipo de parto), fatores de risco gestacional (hemorragia, deslocamento prematuro de placenta, placenta prévia, amniorrexe prematura, oligoddrâminio, TORCHS, infecção do trato urinário, alcoolismo, diabetes, hipertensão, doença hipertensiva específica da gestação, tabagismo, uso de medicações e drogas) e parto (apresentação e tipo de parto).
Tratando-se de investigação, cuja fonte de dados foi a declaração de nascidos vivos e os prontuários das gestantes, foram adotados nomes fictícios de pessoas, para cada protocolo preenchido, visando evitar que em algum momento os participantes fossem identificados e que pudessem vir a sofrer prejuízos de qualquer natureza oriundos deste estudo. E por tratar-se também de base de dados secundária, não foi utilizado termo de compromisso livre e esclarecido.

Os dados (grupo-caso) foram coletados na primeira via de declarações de nascidos vivos que fica arquivada no Departamento de Vigilância Epidemiológica da Secretaria Municipal de Saúde. E os fatores de risco gestacional (hipertensão arterial, amniorrexe prematura, infecção urinária e oligodramnio), nos prontuários das gestantes no hospital público onde foi realizado o parto. Já os dados do grupo-controle foram recrutados de forma aleatória através da análise do relatório do faturamento hospitalar contendo a relação de todos os partos ocorridos no período do estudo; foram coletados exclusivamente nos prontuários das gestantes e terceira via da declaração de nascidos vivos que fica arquivada no prontuário. A coleta de dados das declarações de nascidos vivos do grupo-caso ocorreu em local diferente daquele do grupo-controle porque a Vigilância Epidemiológica Municipal tem um arquivo das declarações de nascidos vivos prematuros e de baixo peso separado das demais. Essa separação facilitou a identificação dos casos de prematuridade e baixo peso ao nascer.

Para definição dos casos de hipertensão foi adotada a condição clínica em que a pressão arterial sistólica encontrava-se em níveis $\geq 140 \mathrm{mmHg}$ e/ou a pressão diastólica com valores $\geq 90 \mathrm{mmHg}$ ou diagnóstico de hipertensão registrado no prontuário, independentemente do tipo de síndrome hipertensiva (hipertensão crônica, pré-eclampsia, eclampsia e pré-eclâmpsia sobreposta à hipertensão crônica).

Foram excluídas do estudo as gestantes que tiveram filhos com anomalias congênitas (cinco no grupo-caso) e aquelas cujo prontuário não foi encontrado (seis no grupo-caso) por não haver informação sobre os fatores de risco gestacional. A variável número de consultas de pré-natal (mediana $=7$ ) foi dicotomizada.

ANÁLISE ESTATÍSTICA

As associações das possíveis variáveis preditoras da prematuridade e baixo peso ao nasce foram analisadas por meio de modelos hierarquizados de regressão logística múltipla, pelo procedimento PROC GENMOD do programa SAS Institute Inc., Cary, NC, USA, Release 9.2, 2010.

$\mathrm{Na}$ hierarquização do modelo, as variáveis foram agrupadas em três blocos: distal (idade da gestante, sexo do recém-nascido, situação conjugal, escolaridade e tipo de parto), mesial (número de consultas de pré-natal e tipo de gravidez) e proximal (risco gestacional). Foram testadas no modelo de regressão logística múltipla as variáveis com nível de significância de $p \leq 0.20$ de cada bloco, permanecendo no modelo aquelas que continuaram associadas ao desfecho com $p \leq .05$ após o ajuste para as variáveis do mesmo bloco e para as hierarquicamente superiores. 


\section{RESULTADOS}

A prevalência de gestantes com hipertensão arterial durante a internação para o parto, na amostra estudada, foi de $13.5 \%$. A diferença entre esse percentual e o de recém-nascidos de gestantes hipertensas (13.2\%) justifica-se pela ocorrência de 05 gestações duplas.

No quadro 1 observa-se que houve associação significativa $(p<.05)$ entre os fatores considerados de risco gestacional e a prematuridade. Gestantes com hipertensão ou outros fatores de risco gestacional apresentaram maior chance de ter filhos prematuros $(p<.05)$.

Foi observada também associação significativa entre o número de consultas pré-natais e a prematuridade. Gestantes que foram a menos consultas tiveram maior chance de ter filhos prematuros $(p<.05)$ (VER QUADRO 1). 0 tipo de gravidez (única ou dupla) não apresentou associação significativa com a prematuridade $(p>.05)$.

Não se observou associação significativa da idade cronológica da gestante, sexo do recém-nascido, escolaridade e tipo de parto com a prematuridade $(p>.05)$, de acordo com o quadro 1 . As gestantes casadas apresentaram maior chance de ter filho prematuro que as solteiras $(p<.05)$

Gestantes com hipertensão apresentam 3.47 (IC 95\%: 1.37 - 8.81) vezes mais chance de ter filho prematuro que as sem fatores de risco na gestação $(p<.05)$. Gestantes que apresentam outros fatores de risco que não a hipertensão têm 2.21 (IC 95\%: $1.13-4.31$ ) vezes mais chance de ter filho prematuro do que aquelas sem fatores de risco na gestação $(p<.05)$. Gestantes que tiveram menos de sete consultas pré-natais têm $2.31(1.22-4.34)$ vezes mais chance de ter filho prematuro do que as que tiveram pelo menos sete consultas $(p<.05)$, (VER QUADRO 2)

QUADRO 1. Análise individual da associação entre prematuridade e as variáveis dos grupos proximal, mesial e distal

\begin{tabular}{|c|c|c|c|c|c|c|c|}
\hline \multirow[t]{2}{*}{ GRUPO } & \multirow[t]{2}{*}{ VARIÁVEL } & \multirow[t]{2}{*}{ CATEGORIA } & \multirow[t]{2}{*}{$N(\%)^{*}$} & \multicolumn{2}{|c|}{ PREMATURIDADE } & \multirow[t]{2}{*}{$\mathrm{OR}^{*}(\mathrm{IC} 95 \%)$} & \multirow[t]{2}{*}{ P-VALOF } \\
\hline & & & & Sim & Não & & \\
\hline & & & & $N(\%)^{\&}$ & $N(\%)^{\mathbb{2}}$ & & \\
\hline & & Sem & $99(46.7)$ & $24(24.2)$ & $75(75.8)$ & $\operatorname{Ref}^{1}$ & \\
\hline \multirow[t]{2}{*}{ Proximal } & \multirow[t]{2}{*}{ Risco } & Hipertensão & $28(13.2)$ & $14(50.0)$ & $14(50.0)$ & $3.12(1.31-7.47)$ & 0.0104 \\
\hline & & Outros riscos & $85(40.1)$ & $36(42.4)$ & $49(57.6)$ & $2.30(1.22-4.31)$ & 0.0097 \\
\hline \multirow{4}{*}{ Mesial } & \multirow{2}{*}{$\begin{array}{l}\text { Consulta } \\
\text { Pré natal }\end{array}$} & $<7^{5}$ & $90(42.9)$ & $40(44.4)$ & $50(55.6)$ & $2.02(1.14-3.60)$ & 0.0163 \\
\hline & & 7 & $120(57.1)$ & $34(28.3)$ & $86(71.7)$ & $\operatorname{Ref}^{1}$ & \\
\hline & \multirow{2}{*}{$\begin{array}{l}\text { Tipo de } \\
\text { Gravidez }\end{array}$} & Dupla & $10(4.7)$ & $2(20.0)$ & $8(80.0)$ & $\operatorname{Ref}^{1}$ & \\
\hline & & Única & $202(95.3)$ & $72(35.6)$ & $\begin{array}{l}130 \\
(64.4)\end{array}$ & $2.22(0.56-10.71)$ & 0.3226 \\
\hline
\end{tabular}

\begin{tabular}{|c|c|c|c|c|c|c|c|}
\hline & \multirow{3}{*}{$\begin{array}{l}\text { Idade da } \\
\text { Gestante }\end{array}$} & $<18$ anos & $31(14.6)$ & $10(32.3)$ & $21(67.7)$ & $\operatorname{Ref}^{1}$ & \\
\hline \multirow{17}{*}{ Distal } & & 19-34 anos & $158(74.5)$ & $27(36.1)$ & $\begin{array}{l}101 \\
(63.9) \\
\end{array}$ & $1.18(0.52-2.69)$ & 0.6848 \\
\hline & & $>34$ anos & $23(10.8)$ & $7(30.4)$ & $16(69.6)$ & $0.92(0.28-2.94)$ & 0.8866 \\
\hline & \multirow{2}{*}{ Sexo do RN } & Feminino & $95(44.8)$ & $27(28.4)$ & $68(71.6)$ & $\operatorname{Ref}^{1}$ & \\
\hline & & Masculino & $117(55.2)$ & $47(40.2)$ & $70(59.8)$ & $1.69(0.95-3.02)$ & 0.0754 \\
\hline & \multirow{5}{*}{$\begin{array}{l}\text { Situação } \\
\text { Conjugal }\end{array}$} & Solteira & $62(29.5)$ & $16(25.8)$ & $46(74.2)$ & $\operatorname{Ref}^{1}$ & \\
\hline & & Casada & $88(41.9)$ & $41(46.6)$ & $47(53.4)$ & $2.51(1.24-5.08)$ & 0.0107 \\
\hline & & União estável & $56(26.7)$ & $15(26.8)$ & $41(73.2)$ & $1.05(0.46-2.39)$ & 0.9039 \\
\hline & & Divorciada & $1(0.5)$ & $0(0.0)$ & $1(100.0)$ & - & \\
\hline & & $\overline{V i u ́ v a}$ & $3(1.4)$ & $1(33.3)$ & $2(66.7)$ & $1.44(0.12-16.94)$ & 0.07731 \\
\hline & \multirow{6}{*}{ Escolaridade } & Sem & $4(1.9)$ & $1(25.0)$ & $3(75.0)$ & $\operatorname{Ref}^{1}$ & \\
\hline & & Fundamental I & $28(13.2)$ & $3(10.7)$ & $25(89.3)$ & $0.36(0.03-4.66)$ & 0.4342 \\
\hline & & Fundamental.II & $67(31.6)$ & $25(37.3)$ & $42(62.7)$ & $1.79(0.18-18.11)$ & 0.6238 \\
\hline & & Médio & $96(45.3)$ & $35(36.5)$ & $61(63.5)$ & $1.72(0.17-17.19)$ & 0.6437 \\
\hline & & Superior incop & $2(0.9)$ & $1(50.0)$ & $1(50.0)$ & $3.00(0.08-107.45)$ & 0.5474 \\
\hline & & Superior comp & $15(7.1)$ & $9(60.0)$ & $6(40.0)$ & $4.50(0.37-54.16)$ & 0.2360 \\
\hline & \multirow{2}{*}{ Tipo de parto } & Cesáreo & $75(35.4)$ & $31(41.3)$ & $44(58.7)$ & $1.54(0.86-2.76)$ & 0.1474 \\
\hline & & Vaginal & $137(64.6)$ & $43(31.4)$ & $94(68.6)$ & $\operatorname{Ref}^{1}$ & \\
\hline
\end{tabular}

"Porcentagem do total; ${ }^{8}$ Porcentagem na linha; ${ }^{*}$ Odds Ratio bruto e intervalo de confiança; ${ }^{2}$ Nível de referência

QUADRO 2 Resultado da análise regressão logística múltipla para associação entre prematuridade e variáveis avaliadas

\begin{tabular}{lcccc}
\hline VARIÁVEL & CATEGORIA & PREMATURIDADE & OR $^{\star}($ IC95\%) & P-VALOR \\
\hline \multicolumn{5}{c}{${\mathrm{N}(\%)^{8}}^{8}$} \\
\hline Risco & Sem & $24(24.2)$ & Ref $^{1}$ \\
\hline & Hipertensão & $14(50.0)$ & $3.47(1.37-8.81)$ & 0.0104 \\
\hline & Outros riscos & $36(42.4)$ & $2.21(1.13-4.31)$ & 0.0097 \\
\hline Consulta & $<7^{5}$ & $40(44.4)$ & $2.31(1.22-4.34)$ & 0.0163 \\
\hline Pré natal & 7 & $34(28.3)$ & Ref $^{1}$ & \\
\hline
\end{tabular}

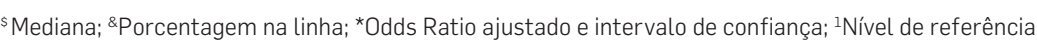

Entre os outros fatores de risco gestacional, os mais comuns foram amniorrexe prematura (18.4\%), infecção urinária (11.8\%) e oligodramnio (8.5\%).

Observou-se associação significativa do risco gestacional e a prematuridade com o baixo peso do recém-nascido $(p<.05)$. 0 baixo peso do recém-nascido não apresentou associação significativa com o número de consultas de pré-natal e o tipo de gravidez $(p>.05)$. 0 baixo peso do recém-nascido não apresentou associação significativa com a idade da gestante, sexo do recém-nascido, situação conjugal, escolaridade e tipo de parto ( $p>.05$ ) (QUADRO 3). 
a mortalidade neonatal (Sampaio, Pinto, \& Sampaio, 2012). Segundo os achados do presente estudo, tal condição foi associada à hipertensão arterial durante a gestação, bem como com o número de consultas de pré-natal.

É uma complicação frequente das síndromes hipertensivas, seja em decorrência de trabalho de parto espontâneo ou pela interrupção da gravidez por indicação obstétrica devido ao comprometimento materno-fetal (Ministério da Saúde, 2012b).

Os prematuros têm risco elevado de adoecer e morrer devido ao seu incompleto desenvolvimento fetal e maior susceptibilidade às infecções complicadas pela manipulação e grande tempo de permanência nas unidades neonatais (Teixeira, Vasconcelos, \& Ribeiro, 2015). Além disso, a prematuridade também pode influenciar a qualidade de vida consequente às possíveis sequelas, o que exige políticas públicas que possam dar suporte a esses recém-nascidos. Desse modo, verifica-se a seriedade das consequências da prematuridade nas gestações advindas de mulheres com hipertensão arterial.

A prevalência de gestantes com hipertensão arterial mostrada no estudo encontra-se em consonância com o estudo realizado no Município de São Paulo, onde $13.9 \%$ das gestantes foram diagnosticadas com hipertensão induzida ou agravada pela gestação (Chaim, Oliveira, \& Kimura, 2008); superior aos $8.4 \%$ encontrados nas gestantes atendidas no município do Rio de Janeiro (Vettore et al., 2011), e inferior aos $18.4 \%$ encontrados no estudo realizado no Hospital Universitário localizado no município de Maceió-AL (Soares, Cavalcante, Santos, França, \& Junior, 2015). É importante considerar que o presente estudo foi realizado em unidade hospitalar de referência para o atendimento de gestantes de alto risco, fator que pode influenciar a prevalência de gestantes com hipertensão arterial.

A hipertensão arterial crônica durante a gestação pode levar a complicações maternas e perinatais, como sobreposição de pré-eclâmpsia, restrição do crescimento fetal, prematuridade, deslocamento prematuro de placenta, morte fetal e maior risco de morte materna (Sass, Santos, Atallah, \& Camano, 2002).

Outro fator associado à prematuridade encontrado diz respeito ao número de consultas de pré-natal. Observou-se que as gestantes com menos de sete consultas apresentaram maior chance de ter filho prematuro do que as com pelo menos sete consultas. Segundo recomendações do Ministério da Saúde, um número mínimo de seis consultas de pré-natal deve ser realizado pelas gestantes que não apresentem fatores de risco detectados na primeira consulta (Ministério da Saúde, 2012c).

O pré-natal é amplamente reconhecido como um dos principais determinantes da evolução gestacional normal (Pedraza, 2016) e deve ser iniciado o mais cedo possível para fortalecer a adesão da gestante ao acompanhamento e possibilitar a detecção e prevenção de complicações obstétricas. Segundo Chaim et al. (2008), a assistência pré-natal desempenha um papel de extrema importância na prevenção da morbidade e mortalidade materna e perinatal, uma vez que a hipertensão arterial na gravidez ainda não pode ser evitada, mas o óbito materno pode ser impedido.

\section{DISCUSSÃOO}

A prematuridade ainda é considerada como elevada em alguns municípios brasileiros, 0 que representa um problema a ser enfrentado no país, considerando sua inter-relação com 
A realização do pré-natal pelas gestantes é uma das atividades da atenção primária à saúde da mulher que visa evitar que problemas durante a gestação interfiram na qualidade de vida tanto da mulher quanto do neonato. Dessa forma, o pré-natal propicia à gestante um cuidado maior com a própria saúde e adoção de hábitos de vida saudáveis para si e para seu filho. Assim, orientações quanto aos hábitos alimentares, atividade física e mudanças no comportamento sedentário são de extrema valia para modificar a prevalência de intercorrências durante a gestação, no parto e pós-parto.

Analisando-se a relação entre prematuridade e variáveis do grupo distal avaliadas (idade da gestante, sexo do RN, situação conjugal, escolaridade e tipo de parto) não foram encontradas associações estatisticamente significantes, embora outros estudos tenham mostrado associação para pelo menos um desses fatores (Aragão et al., 2004; Cascaes, Gauche, Baramarchi, Borges, \& Peres 2008; Sampaio et al., 2012).

Outro desfecho avaliado foi o baixo peso ao nascer, sendo encontrada associação com hipertensão arterial e prematuridade. 0 baixo peso ao nascer sofre influência direta da duração da gestação, portanto o recém-nascido prematuro está mais propenso a ter baixo peso que os não prematuros. Neste estudo observou-se que a prematuridade esteve associada com o baixo peso, corroborando um estudo realizado nos municípios de Juazeiro e Petrolina (Figueredo, 2015). 0 ganho ponderal é maior no terceiro trimestre da gestação, em especial, a partir de 34 semanas, o que ajuda a compreender a estreita relação entre prematuridade e baixo peso ao nascer (Silva, 2012). Deve-se, portanto, priorizar ações para evitar os partos prematuros, através de medidas sistematizadas que visem reduzir a frequência de baixo peso ao nascer.

Tal achado quanto â existência de associação com significância estatística entre hipertensão arterial e baixo peso ao nascer também foi registrado por Rompinelli, Massaro, Utyama, Freitas e Guidoni (2016) e Figueiredo et al. (2015). Quanto mais precoce o diag nóstico da hipertensão arterial somado à intervenção em momento oportuno, maiores são as possiblidades de se conduzir uma gestação sem complicações maternas e agravos do concepto. Essa doença determina o baixo peso ao nascer, visto que pode acelerar o trabatho de parto, causando, assim, a prematuridade e o baixo peso ao nascer (Jesse, Swanson, Newton, \& Morrow, 2009; Silva, Almeida, Matsuo, \& Soares, 2009).

No presente estudo o baixo peso do recém-nascido não apresentou associação significativa com o número de consultas de pré-natal. Em contradição, um estudo realizado em Londrina/PR, entre 2000 e 2009 , apontou que $65.9 \%$ das mulheres que realizaram até seis consultas de pré-natal evoluíram para o parto prematuro extremo (menor que ou igual a 31 semanas de gestação) e $51.7 \%$ dos bebês nasceram com menos de $1000 \mathrm{~g}$. Entretanto, entre as mulheres que realizaram mais de sete consultas, foi menos frequente o parto prematuro extremo e o baixo peso (Ferrari, Bertolozzi, Dalmas, \& Girotto, 2014).

Outros fatores de risco gestacional, que não a hipertensão, também foram encontrados. Dentre os mais frequentes estão a amniorrexe prematura, a infecção urinária e o oligod- râmnio. Gestantes que apresentaram esses fatores de risco mostraram-se mais propensas ao parto prematuro

A amniorrexe prematura se relacionou com $18,4 \%$ das gestações. Em estudo realizado num hospital público do município de Fortaleza, observou-se que cerca de 2,6 a 14,4\% das gestantes apresentaram essa complicação (Costa et al., 2014), que se constitui em causa importante de partos prematuros (cerca de $1 / 3$ dos casos), o que contribui para aumento da mortalidade perinatal. Pode influenciar também a mortalidade materna pelos riscos de infecção (Ministério da Saúde, 2012b). Dentre as complicações que esse fator de risco pode induzir no recém-nascido, encontram-se a prematuridade, a síndrome do desconforto respiratório e sepses (Al-Riyami, Al-Shezawi, Al-Ruheili, Al-Dughaishi, \& Al-Khabori, 2013).

A infecção urinária foi encontrada em $11.8 \%$ das gestantes. Percentuais maiores são descritos na literatura que mostra essa ocorrência em 17 a $20 \%$ das gestações e sua associação a complicações, como rotura prematura de membranas ovulares, trabalho de parto prematuro, corioamnionite, febre no pós-parto, sepse materna e infecção neonatal (Ministério da Saúde, 2012b).

O oligo-hidrâmnio esteve presente em $8.5 \%$ das gestações, percentual acima do encontrado na literatura sobre o assunto, que mostra que cerca de 0.5 a $5 \%$ das gestações apresentam esse fator de risco. A oligo-hidramnia aumenta o risco de compressão funicular e se associa com frequência ao sofrimento fetal (Ministério da Saúde, 2012b).

Baseados nas evidências encontradas neste estudo, estratégias na atenção primária à saúde devem ser estabelecidas, visando a reduzir de modo eficiente os riscos maternos e perinatais. Dentre essas estratégias, destaca-se o oferecimento de pré-natal de baixo risco de qualidade, para identificação de fatores de risco gestacional, dentre eles a hipertensão arterial.

Uma vez diagnosticada a hipertensão, a gestante deve ser encaminhada e assegurada do acompanhamento de alto risco na unidade de referência, em parceria com a unidade de saúde da família, porque, quanto mais precoce for esse diagnóstico, somado à conduta em momento oportuno, maiores as possibilidades de conduzir uma gestação sem complicações maternas e perinatais.

Além disso, o incentivo, a orientação e a promoção da alimentação saudável, da atividade física e das práticas corporais no âmbito da atenção primária à saúde da gestante devem ser ações constantes do profissional de saúde, a fim de tornar o dia a dia da gestante mais ativo, contribuir para o crescimento do feto e diminuir o risco de parto prematuro (Ministério da Saúde, 2012c).

A prática de exercícios físicos orientados e acompanhados por um profissional de educação física especializado, juntamente com acompanhamento médico e de enfermagem, é recomendada, uma vez que essa prática tem como meta manter a capacidade cardiorrespiratória e o condicionamento físico ao longo do processo, além de auxiliar na prevenção e no controle do diabetes gestacional, do ganho de peso materno e da hipertensão gestacional (Nascimento, Surita, \& Cecatti 2012; Zavorsky \& Longo, 2011). 
Dentre outros benefícios proporcionados por meio do exercício físico durante a gestação, destacam-se: a melhora da postura, quando a mulher suporta melhor seu peso corporal e melhora o equilíbrio; a prevenção de doenças cardiovasculares, principalmente a hipertensão arterial crônica; a manutenção do peso; o equilíbrio dos níveis glicêmicos; a melhora da autoestima; maior controle na musculatura do assoalho pélvico, oferecendo maior suporte ao feto e ainda prevenindo a ocorrência de incontinência urinária; melhor controle dos níveis pressóricos (Giacopini, Oliveira, \& Araújo, 2016; Rezende, Marchiori, \& Teodoro, 2016).

\section{CONCLUSÕES}

Devido à falta de informação nos prontuários das gestantes sobre a classificação dos tipos de síndrome hipertensiva e complexidade do diagnóstico diferencial, não foi possível fazer a análise separadamente. Assim, os dados foram analisados considerando hipertensão arterial durante a internação para o parto, independentemente do tipo.

$\mathrm{E}$, apesar das limitações das informações disponíveis nas declarações de nascidos vivos e prontuários das gestantes, seu uso possibilitou identificar que houve associação significativa da hipertensão arterial durante a internação para o parto com a prematuridade e o baixo peso ao nascer.

Sendo assim, esses resultados servirão para reforçar a necessidade de implementação de políticas públicas de promoção de saúde materno-infantil não só no município estudado, mas em nível regional, com oferecimento de seguimento pré-natal diferenciado e com melhor qualidade na atenção primária à saúde e planejamento de medidas de prevenção, identificação e controle da doença, bem como o encaminhamento da gestante hipertensa ou com outros fatores de risco gestacional em tempo hábil para o pré-natal de alto risco e a garantia do atendimento dessas gestantes na atenção secundária e terciária.

Além disso, faz-se necessária também a vigilância das complicações neonatais e o seguimento desses recém-nascidos para o entendimento das repercussões da hipertensão durante a gravidez no desenvolvimento e qualidade de vida das crianças.

\section{FONTES DE FINANCIAMENTO}

\section{E AGRADECIMENTOS}

A Universidade do Estado da Bahia (UNEB) pelo financiamento deste estudo, por meio da Bolsa PAC.
Al-Riyami, N., Al-Shezawi, F., Al-Ruheili, I., Al-Dughaishi, T., \& Al-Khabori, M. P. (2013). Fatores de risco para prematuridade em São Luís, Maranhão, Brasil erinatal outcome in pregnancies with extreme preterm premature rupture of membranes (Mid-Trimester PROM). Sultan Qaboos University Medical Journal, 13(1), 38-42

Aragão, V. M. F., Silva, A. A. M., Aragão, L. F., Barbieri, M. A Bettiol, H., Coimbra, L. C., \& Ribeiro, V. S. (2004). Fatores de risco para prematuridade em São Luís, Maranhão, Brasi Cadernos de Saúde Pública, 20(1), 57-63.

Cascaes, A. M. Gauche, H. Baramarchi, F. M. Borges, C. M. \& Peres, K. G. (2008). Prematurida e fotores C.M. \& Peres, K. G. (2008). Preneturide de 2005: Análise dos dados do Sistema de Informações sobre Nascidos Vivos. Cadernos de Saúde Pública 24(5), 1024-1032.

Chaim, S. R. P., Oliveira, S. M. J. V., \& Kimura, A. F. (2008). Hipertensão arterial na gestação e condições neonatais ao nascimento Acta Paulista de Enfermagem 21 (1) 53-58. Costa, G. M. Tibúrcio, J. D. Olivera V. C. Gontjo, \& Guimarães, E. A. A. (2014). Determinantes do baixo peso ao nascer a partir das declarações de nascidos vivos. Ciência e Enfermeria, XX(3), 21-31.

Ferrari, R. A. P., Bertolozi, M. R., Dalmas, J. C., \& Girotto, E. (2014). Associação entre assistência pré-natal e mortes neonatais, 2000-2009, Londrina-PR. Revista Brasilera de Enfermagem, Brasilia, 67 (3), 354-359.

rade Enedo A. M. G Cruz, S. S. Gomes Fitho I S Souza,

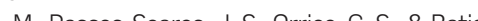
(2015). Fatores de risco do baixo peso ao nascer nos munćpios de Juazeiro (BA) e Petrolina (PE). Revista de Ciências Médicas e Biológicas, 14(1), 22-29.

Giacopini, S. M., Oliveira, D. V., \& Araújo, A. P. S. (2016) Benefícios e recomendações da prática de exercicios físicos na gestação. Revista BioSalus, 1(1), 1-19.

Jesse, D. E., Swanson, M. S., Newton, E. R., \& Morrow J. (2009). Racial disparities in biopsychosocial factors and spontaneous preterm birth among rural low-income women. Journal of Midwifery Womens Health, 54(1) 35-42. Ministério da Saúde. (2012a). Gestação de alto risco: Manual técnico (5a Ed). Brasília.

Ministério da Saúde. (2012b ). Saúde Brasil 2011: Uma análise da situação de saúde e a vigilância da saúde da mulher Brasilia: MS. Mortalidade Materna no Brasi: Principais causas de morte e tendências temporais no período de 1990 a 2010

Ministério da Saúde. (2012c). Secretaria de atenção à Saúde. Departamento de Atenção Básica, noํ32. Atenção ao pré-natal de baixo risco. Brasília: Editora do Ministério da Saúde.
Ministério da Saúde. (2014). Datasus. Cadastro no CNES. Secretaria de Atenção a Saúde.

Nascimento, S. L., Surita, F. G., \& Cecatti, J. G. (2012). Physical exercise during pregnancy: A systematic review. Current Opinion in Obstetrics and Gynecology 24(6), 387-394,.

Pedrazza, D. F. (2016). Fatores associados ao baixo peso ao nascer em cidades da Paraíba. Revista Atenção à Saúde, 14(47), 42-48.

Rezende, R. S. Marchiori. T. P. \& Teodoro, E. C. M. -eclâmpsia. Revista Brasileira de Fisiologia do Exercicio, 15(1), 36-45.

Rompinelli, V. O., Massaro, N., Utyama, J. A., Freitas, N. M. M., \& Guidoni, R. G. R. (2016). 0 impacto dos fatores de risco materno na estratificação de recém-nascido baixo peso. Revista UNILUS Ensino e Pesquisa, 13(30), 54-63. Sampaio, R. M. M., Pinto, F. J. M., \& Sampaio, J. C. (2012). Fatores de risco associados à prematuride Pu vivos no estado do Ceara Remista Bada em nascidos vivos no estado do Cear (1) 969-978.

Sass, N., Santos, J. F. K., Atallah, A. N., \& Camano, L. (2002). Hipertensão arterial crônica leve e moderada na gravidez: Práticas terapêuticas baseadas em evidências. Femina, 30(7), 449-453.

Silva, A. M. R., Almeida, M. F., Matsuo, T., \& Soares, D. A. (2009). Fatores de risco para nascimentos pré-termo ca, 25(10), 2125-2138.

Silva, T. R. S. R. (2012). Nonbiological maternal risk factor for low birth weight on Latin America: A systematic review of literature with meta-analysis. Einstein, 10(3), 380-385.

Soares, J. C. S., Cavalcante, A. L., Santos, L. G. M. L. França, A. M. B. \& Junior A. F. S. X (2015) Obitos maternos por síndromes hipertensivas induzidas pela gravidez no estado de Alagoas no período de 2008-2013. Ciências Biológicas e da Saúde, 2(3), 67-79.

Teixeira, A. L., Vasconcelos, L. D., \& Ribeiro, R. A. F. (2015). Prevalência de patologias e relação com a prematuridade em gestação de alto risco. Revista $\mathrm{Cl}$ ências em Saúde, 5(4).

Vettore, M. V., Dias, M., Madeira, R. M. S. D., Vettore, M. V. \& Leal. M. C. (2011). Cuidados pré-natais e avaliação do manejo da hipertensão do SUS no município do Rio de Janeiro, Brasil. Cadernos de Saúde Pública, 27(5), 1021-1034.

Zavorsky, G. S., \& Longo, L. D. (2011). Exercise guidelines in pregnancy: New perspectives. Sports Medicine $41(5), 345-360$. 